\title{
Better Health Care Through Technology
}

Mache Creeger, Emergent Technology Associates

everaging technology to support aging relatives in their homes is a cost-efficient way to maintain health and happiness and extend life. As the technology expert for my extended family, it has fallen to me to architect the infrastructure that will support my family's aging loved ones in their homes as long as possible. Over the years, I have assisted four different senior households in achieving this goal, and although things have been bumpy at times, I have refined technical solutions and methodologies that seem to work well.

There are two major elements involved here: the prompt response to a medical emergency and the consolidation and timely dissemination of current medical information. Here is some of what I have learned.

\section{DAILY LIVING AT HOME}

In my particular care experiences, loved ones are either living alone or with a spouse. In an emergency, the spouse or patient must call for help. If the spouse is not present, then it is up to the patient to make the call. This can be a problem if the type of distress being experienced prevents the patient from using the telephone.

I recommend the installation of a medical alerting system that uses the telephone to contact a 24/7 emergency call center. ${ }^{1}$ Alert units typically have a base station that operates much as a powered speaker phone with RJ45 and RJ11 connectors in the rear. The base station is usually placed in the bedroom, connected to a RJ31-X device, and typically has a large button to be depressed in an emergency. The device also includes a waterproof medallion with an emergency button that can communicate via RF signal to the base station within the confines of the home. This provides coverage throughout the house, even while the user is bathing. Additionally, you can configure these units so they require some type of activity by the user during every 12- or 24-hour period.

A successful alert-unit installation requires that you, or a third-party installer, modify the home telephone wiring. A new pair of wires needs to be installed to take the telephone line from the point at which the telephone service enters the house (NID/DMark) directly and only to the RJ31-X in the bedroom. From the RJ31-X, a second

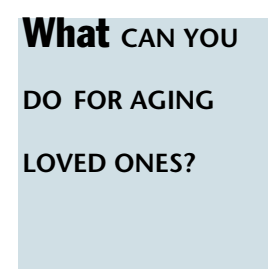

pair of wires then takes the telephone line back to the NID/DMark, linking up with the wiring that drives the rest of the

telephones in the house. Either CAT3 or CAT5 cable can be used to link the RJ31-X with the NID/DMark. In this way, even if all the telephone extensions in the house are off their hooks (a fairly common occurrence among the hearing impaired-affecting many older people), when the emergency button is depressed, the alert unit cuts off all telephones beyond the unit, obtains a dial tone, and contacts the emergency call center.

The RJ31-X has a single eight-pin RJ45 connector, of which four pins are used. It allows for the insertion of a telephonic device, in series, into an existing two-wire telephone line (represented by wires labeled TIP and RING). The medical-alert device is connected to the RJ31-X via an RJ45 patch cable placing the device in series between the NID/DMark and the rest of the telephones in the house. Often the alert device has an extra RJ11 connector that serves as an additional home telephone extension.

Pins 4 (RING) and 5 (TIP) on the RJ31-X represent the telephone line coming from the NID/DMark to the alert device, and pins 1 (RING) and 8 (TIP) represent the telephone line coming from the alert device and going to the other telephones in the house. Removal of the RJ45 patch cable from the RJ31-X causes two independent spring-loaded shorting bars to short pins 1 through 4 and separately short pins 5 through 8 . This allows the removal of the alert device from the telephone line without disturbing its integrity from the NID/DMark to the other telephones in the house.

\section{WHEN THINGS GO BUMP}

I have been doing this for about 15 years, so I can say with confidence that you should count on things not always going smoothly. You are trying to project a technical solution over distance to a person who is typically not technically sophisticated and has some measure of chronic illnesses and/or frailty. You are going to have problems. 
When I first started working with alert units, they were supported by volunteer members from the local community. These people were typically retired, giving back to their community, but had no technical background and minimal or no training. On one occasion an older volunteer visited my octogenarian father-in-law at home in Pennsylvania to see how he and the alert unit were getting along. To be helpful, he replaced the four-pair RJ45 patch cable that connected the alert unit to the RJ31-X with a standard two-pair RJ11 telephone extension cable. He did not tell anyone about this kind act before he left.

Soon afterward, I received a phone call in California from my father-in-law using a neighbor's phone informing me that all the telephones in his house were dead. After several hours struggling with my father-in-law over the neighbor's phone, I solved the riddle. A call to the volunteer confirmed my diagnosis and revealed that the original RJ45 patch cable had since been thrown away.

\section{Installing Webcams and audio}

in the home would make videoconferencing available to multiple caregivers.

After many phone calls around my father-in-law's small Pennsylvania town, I finally tracked down an exorbitantly priced RJ45 patch cable and had my father-in-law purchase it. Once he plugged it in, everything started working again.

\section{TIMELY DISSEMINATION OF MEDICAL INFORMATION}

Many seniors live with chronic medical conditions that get worse over time. This usually means they have multiple doctors, including a PCP (primary care physician) and an increasing number of specialists. As a result, medical records-in most cases on paper-are typically spread out across a number of medical offices. In a perfect world, as a person's medical condition changes, the PCP would coordinate consultations with other specialists to achieve an optimal care plan that addresses all the patient's illnesses and constraints. We don't live in a perfect world, however, and in my experience doctors tend to focus on their specialty, with collaboration the exception rather than the rule.

Most of the people I help are in their 80s or older, ill, somewhat frail, hearing-impaired, and are not sophisticated consumers of medical services. When they go to a physician for the first time, the doctor or nurse struggles to extract a viable medical history. Critical issues are often omitted or incorrectly captured. Extending health and subsequently life in the face of increasing complex illness requires efficient capture and management of medical information. To address this deficiency, I integrate all pertinent medical facts into a single set of summary documents. The patient walks in, hands the doctor the summary, and then waits for any questions. If the job is done right, the only questions will be about the patient's current health status.

As technology specialists, we understand the importance of making all constraints visible when fashioning an effective engineering solution to a problem. I have found the same axiom to be true in long-term medical care. As a result, I have encouraged my family members to take a small step toward managing their medical records directly and recommend that three documents be prepared and maintained: an up-to-date medication list, a brief medical history covering major chronic illnesses, and the name, specialty, address, and telephone numbers of all physicians. After these documents are prepared, I have them reviewed by the pertinent doctors for clarity and completeness, and I continuously update them in electronic form with an associated revision date.

Accurate information is critical for survival, especially in emergency situations. Trips to the emergency room can become a frequent occurrence for the elderly, and by definition, the patient is distressed and a complete medical history may be unavailable.

For these reasons, I keep the three documents electronically accessible on a computer with a fax modem (fax seems to be the lingua franca of the medical community). As a technology professional, I would like to take advantage of more advanced communication pathways such as Web sites, e-mail, and/or Web services integration to computerized patient records, but don't even think about it. Most private doctors' offices work only with paper charts and accept only faxes because they can be put directly into the patient chart with no intermediate steps. If they have computers at all, it's probably only for managing appointments and billing. Internet access is often avoided as it is looked upon at best as a staff diversion and at worst as a security risk.

In providing information to an ER, you have to consider that the treating physician wants only to have a piece of paper in hand providing the key information needed to get the patient stable. Although ERs have 
computers, e-mail accounts are usually not accessible, and staff is discouraged from using Internet browsers. As for integrating into computerized patient record systems, even if the hospital had one, which is doubtful, I believe it is way too early to expect it to accept any information from outside the institution.

Therefore, the best action to take is to fax summaries directly to the hospital to support the attending physician's efforts with the most complete medical information available. I have been told on many occasions after supplying the documents in this way that the information was extremely helpful in delivering fast, high-quality care to alleviate the distress.

\section{THINGS TO THINK ABOUT FOR THE FUTURE?}

With an always-on broadband Internet connection, caregivers can maintain a virtual presence with a loved one through existing videoconferencing applications. Installing Webcams and audio in the home would make videoconferencing available to multiple caregivers and allow family members to share day-to-day experiences with the loved one without regard to physical distance. ASPs (application service providers) may spring up to offload medical information capture and dissemination from the caregiver to a subscription service.
As people age and have to deal with increasingly complex chronic illnesses, the care challenge is similar to juggling an ever-increasing number of balls. Although we can never handle an infinite number of balls, we can certainly handle more with effective use of technology and some simple methodologies. With that perspective, you can increase the health and overall quality of life for the people you love for as long as is reasonable. If you can achieve that, you have done yourself, your family, and your loved ones a great service.

\section{REFERENCE}

1. I have used two alert-device vendors and found them both satisfactory: Rescue Alert (http://www.rescuealert. com) and Lifeline (http://www.lifelinesys.com).

\section{LOVE IT, HATE IT? LET US KNOW}

feedback@acmqueue.com or www.acmqueue.com/forums

MACHE CREEGER (mache@creeger.com) is a long-time technology industry veteran based in Silicon Valley. He is the principal of Emergent Technology Associates, marketing and business development consultants to technology companies worldwide.

() 2006 ACM 1542-7730/06/1100 $\$ 5.00$ 